\title{
Clases online o la necesidad de adaptación a una nueva forma de establecer el proceso de enseñanza-aprendizaje
}

\author{
Online Classes or the Need to Adapt to a New Way of Establishing the \\ Teaching-Learning Process
}

Tomás Soto-Aguirre ${ }^{1}$

\begin{abstract}
RESUMEN
La pandemia mundial ha obligado a la implementación de clases online. En Chile, la premura ha invisibilizado la dispar realidad de conocimientos tecnológicos de los y las docentes y la escasa variedad de estrategias para establecer una nueva manera de transmitir su saber. El confinamiento se vuelve una oportunidad para realizar los cambios que la educación chilena necesita en este ámbito.
\end{abstract}

Palabras claves: avance tecnológico; innovaciones educativas; clases online; estrategias docentes; brecha tecnológica.

\begin{abstract}
The world pandemic situation has forced the implementation of online classes. In Chile, the urgency has made invisible the unequal amount of technological knowledge between teachers, and the limited variety of strategies to establish a new way of transmitting their knowledge. This lockdown becomes an opportunity to make the changes that Chilean education needs in this field.
\end{abstract}

Keywords: Technological advance; educational innovations; online classes; teaching strategies; technological gap.

\footnotetext{
1 Académico, Universidad de Las Américas, Viña del Mar, Chile; magíster en Educación; tomas.soto.aguirre@edu.udla.cl
} 


\section{Introducción}

Los procesos educativos en Chile y en el mundo se han visto sensiblemente alterados a partir de la pandemia COVID-19. Este hecho ha paralizado, o por lo menos alterado notablemente, gran parte de las actividades cotidianas de la educación desde su etapa preescolar hasta la superior.

Con la finalidad de dar una cierta continuidad al año estudiantil, un número importante de instituciones educativas ha implementado el sistema de clases online. Esto, que hace apenas un año era un recurso alternativo cuya implementación masiva se pensaba como algo factible en un mediano o largo plazo, tuvo que ser adecuado a la realidad inmediata.

En el caso de Chile, el estallido social del 18 de octubre de 2019 anticipó la posibilidad de un uso que hoy emergió como una plataforma necesaria para prevenir un eventual contagio y, por cierto, para permitir que los y las estudiantes pudieran comenzar el primer semestre 2020.

La educación intermediada por medios virtuales ha permitido iniciar el proceso educativo; sin embargo, cabe preguntarse qué ocurrirá con ella cuando la emergencia de salud pase y las personas puedan nuevamente retomar sus funciones habituales. Este ensayo aborda esa interrogante, es decir, indaga en el cambio sustantivo e irreversible que la sociedad ha experimentado en el ámbito tecnológico y cómo esto debiera determinar ciertos caminos del quehacer educativo pospandemia, tanto en la parte humana como en la técnica. Ello, por la injerencia que los usos tecnológicos tendrán en la cotidianidad humana en tan solo una década más.

\section{El indefectible avance tecnológico en el siglo XXI y su influencia en las interacciones sociales}

Hace tres años, la empresa Dell Technologies emitió un informe respecto del impacto que tendría la tecnología en el mundo para el año 2030. Este estudio permitió, a partir de una proyección de lo que ya existe y cómo se incorporará al mundo laboral, reconocer las probables implicancias que el devenir tecnológico tendrá en todas las áreas del quehacer humano (Dell, 2017). 
Este análisis se ha dado en el contexto mundial de un desarrollo de las ciencias y la tecnología, cuyas dimensiones no deben ser consideradas de un modo superficial. El decurso que ha tomado la sociedad del último siglo a partir del permanente asedio de objetos, cada uno más sorprendente que el otro, ha significado instalar en la existencia humana, dos conceptos: novedad y obsolescencia (Rifkin, 2010).

Por novedad se entiende el deseo permanente de poseer aquello que pareciera sernos útil y que emerge como un objeto sin el cual no podemos estar actualizados/as. La industria tecnológica, para incorporar estos productos, entre otros recursos, ha utilizado la publicidad con toda la base de seducción de que dispone. Ello le ha permitido operar sobre el mercado para generar una necesidad constante de consumir.

Al observar cómo funciona el concepto de novedad en los/as jóvenes, constatamos que, en general, la tecnología es el medio y el objetivo final de sus deseos. Esto por una razón natural: la juventud, en todas las épocas, ha querido desmarcarse de las personas adultas para encontrar su propia identidad. En este caso, parte importante de esta demarcación está asociada a objetos tecnológicos que, entre otras cosas, permiten la interacción social. En este sentido, tanto la cantidad de horas en que los/as jóvenes permanecen conectados/as (más de seis horas promedio) como el uso que dan a esa conexión, busca una disociación del mundo adulto, puesto que básicamente la utilizan en este orden - para chatear, descargar música, compartir memes y ver videos (Injuv, 2019).

Relacionado de manera directa con lo anterior, y casi como una consecuencia lógica, emerge el concepto de obsolescencia. El concepto de obsoleto alude a algo que ha comenzado a quedar en desuso. Esta percepción tiene un componente material, pero también psicológico.

Por una parte, la rapidez de fabricación de objetos tecnológicos hace caer a éstos en una obsolescencia permanente. Es decir, la materialidad comprable y consumible está en una dinámica de permanente cambio. No obstante, desde el punto de vista psicológico, tiene una implicancia aún más profunda, tanto a nivel social como a nivel educativo.

En lo social, como se señaló, estos implementos permiten un distanciamiento del mundo adulto. El deseo de la actual generación joven de establecer fronteras, fundamentalmente con la generación anterior, recibe un apoyo natural desde el mundo de 
la tecnología. El conocimiento tecnológico que las generaciones jóvenes actuales poseen desde la infancia, ha generado una brecha cada vez mayor con los adultos.

La diferencia de conocimiento para el libre acceso y uso de estos objetos entre los/as jóvenes menores de 25 años y las personas adultas mayores de 40 años es tan radical, que se podría decir que ambas generaciones se desenvuelven en mundos paralelos. En este punto considero interesante utilizar el concepto de identidad que propone Raymond Williams (1980), quien clasifica los elementos propios de una sociedad bajo tres paradigmas: lo arcaico, lo residual y lo emergente.

Lo arcaico se piensa como aquello que pertenece al pasado y la sociedad lo asume así, a diferencia de lo residual, que, habiéndose formado en el pasado, aún se halla en actividad. Finalmente aparece lo emergente, que representa los nuevos significados y valores, las nuevas prácticas y relaciones sociales (Williams, 1980).

Puestas en juego las tres categorías, Williams señala que las políticas culturales y educativas que son menos efectivas corresponden a las que se basan en lo arcaico e ignoran lo emergente. El autor señala que esto ocurre puesto que no logran vincular el peso de la historia con los significados recientes (García, 2001). Este punto es particularmente importante, puesto que añade la idea del valor de lo reciente en la constitución de la identidad, independientemente de sus efectos positivos o negativos. La identidad es, así, un elemento integrado al proceso cultural, lo que desmitifica la idea de que toda identidad debe basarse exclusivamente en concepciones tradicionales.

En general, las decisiones gubernamentales se mueven sobre la base de la tradición, puesto que el Estado representa en sí mismo la defensa de esas estructuras. En ese sentido, la visión de mundo del poder tenderá a inclinarse hacia la tradición, es decir, en la nomenclatura de Williams, hacia lo arcaico.

Si se comparan los conceptos de lo arcaico y lo obsoleto, se puede encontrar una gran similitud, lo que permite afirmar que esta segregación entre la generación joven y la adulta, en virtud de los usos tecnológicos, tiene un matiz adicional: llevar la obsolescencia desde los objetos hacia las personas.

La extraordinaria presencia de objetos conectados por señales virtuales, mediante la Internet of Things (IoT) o Internet de las cosas, ha hecho que un porcentaje importante de 
los/as jóvenes sientan que ciertas personas adultas están obsoletas, en relación a su capacidad de intervenir en la sociedad a través de los medios digitales. Nos encontramos, así, ante una doble obsolescencia posible: la de los usos digitales de la generación adulta y la de la generación misma en virtud de su visión de mundo.

El punto no deja de ser determinante, toda vez que las personas adultas estamos en el nivel donde se toman las decisiones. Es decir, las directrices educativas, en su mayoría, son determinadas a nivel nacional desde un paradigma que, para los/as jóvenes, está más cerca de lo arcaico que de lo emergente.

\subsection{Influencia de la tecnología en el mundo de la educación y del trabajo}

En el área laboral, se reconoce que es muy probable que más del $80 \%$ de los trabajos y carreras que hoy existen sean fuertemente modificados, o incluso dejen de existir, en virtud de la intervención del uso de software o de robótica aplicada. Esta afirmación, que se percibe un tanto radical, debe de ser cotejada con el ya antiguo desafío hecho por Gordon Moore y que luego se estableció como la ley de Moore. Este autor, a mediados de la década de los años 60 del siglo pasado, planteó que el avance de la ciencia se duplicaría cada dos años. Esta predicción, de consecuencias tan radicales, se ha venido cumpliendo hasta la segunda década del siglo XXI (Zukerfeld, 2015) y ha sido complementada por numerosos científicos como, por ejemplo, el físico vasco Pedro Echeñique. "Cada dos años se duplica el conocimiento tecnológico", explica el físico, "así que los estudiantes de hoy trabajarán con tecnología que aún no existe y se enfrentarán a problemas que no conocemos" (Echeñique, citado por Fábregas, 2008).

Gran parte de las innovaciones educativas han sido estimuladas por el avance tecnológico. La emergencia de novedades emanadas desde la ciencia rápidamente ha ido buscando un nicho desde donde proyectarse. En este estado de situaciones, la educación ha sido objeto de numerosos aportes que han buscado una modernización del sistema.

La motivación mayor de las instituciones educativas pareciera ser abordar el diametral distanciamiento que se ha producido en las últimas décadas entre el conocimiento tecnológico de los/as estudiantes y el de los/as docentes. Sin embargo, la implementación de este saber, es decir, incorporar a los docentes dentro de programas de actualización 
tecnológica para usos pedagógicos, se ha realizado a un ritmo que no se condice con el avance exponencial de los descubrimientos y novedades en la materia (Sandel, 2007). Poner estos medios al servicio de la educación permite reflexionar sobre ellos, ver en conjunto a dónde nos llevan, hasta dónde podemos ir y hasta dónde no.

Si el sistema educativo, como parte constituyente fundamental de la sociedad, se queda atrás en incorporar los usos tecnológicos al aprendizaje, se transformará en un mero espectador respecto de cómo los otros poderes sociales (económicos, políticos e, incluso, publicitarios) toman las decisiones por él.

En otras palabras, se ha llegado a un punto en que el acceso a la información por medio de usos tecnológicos es mucho más sencillo y frecuente entre el estudiantado que entre los y las docentes. Esta disociación, junto con hacer sentir a los/as estudiantes en una situación de superioridad en el manejo de redes sociales y acceso tecnológico (con todo lo que ello implica), ha hecho que algunos/as docentes de más de cuarenta años sean caricaturizados/as como boomers, aludiendo con este término a aquella generación que objetivamente nació entre 1950 y 1968 y que, subjetivamente, hoy es vista como integrada por personas que poseen una estructura mental rígida, temerosa de todo lo nuevo y despectiva del mundo juvenil (Cadem, 2018).

La estructura educativa, en lo esencial, no ha sufrido demasiadas modificaciones desde que la sociedad europea necesitó obreros con una instrucción mínima para el desarrollo de la primera revolución industrial. Esta realidad, que ya era resistida a mediados de siglo $\mathrm{XX}$, ha sido la base de toda la crítica y cuestionamiento en el mundo intelectual. En la década de los 70, autores como Anthony Platt o Michel Foucault expresaron una visión extremadamente crítica del sistema educativo.

Platt (1977) planteaba la educación como un mecanismo de adiestramiento para la fuerza de trabajo y Foucault (1976) consideraba que el educador, entre otros profesionales, ha reemplazado al verdugo respecto de la corrección y el castigo. Ambos analizaron la educación como una estructura impuesta para generar represión. Esta visión crítica continuó en el siglo XXI con autores como Jeremy Rifkin (2010), quien establece un análisis profundo sobre la ausencia de empatía en la educación tradicional, y Ken Robinson $(2009,2015)$, quien describe al formato escolar como la estructura perfecta para anular la creatividad en los niños. 
CLASES ONLINE O LA NECESIDAD DE ADAPTACIÓN A UNA NUEVA FORMA DE ESTABLECER EL PROCESO DE ENSEÑANZA-APRENDIZAJE / SOTO-AGUIRRE

\subsection{OCDE frente a la intervención de la tecnología en el mundo del trabajo}

Durante 2019, la Organización para la Cooperación de Desarrollo Económico (OCDE) publicó un estudio sobre el futuro del trabajo (OCDE, 2019), en el cual señala a Chile como el cuarto país del mundo en mayor riesgo por la automatización laboral. Este dato por sí solo es una luz de alerta para toda la estructura educativa chilena. Es interesante indagar en cuánto de lo que se está haciendo hoy en educación propende al desarrollo e integración de las habilidades tecnológicas y creativas en los estudiantes del país.

En el marco de dicho estudio, se señala que todos los niveles de la educación deben hacer su aporte para desarrollar las habilidades requeridas por los estudiantes; sin embargo, la velocidad y profundidad de los cambios impiden que sea la educación inicial la que modele estas capacidades.

La educación superior se establece entonces como el último recurso para desarrollar las competencias necesarias que demanda esta transición hacia un futuro laboral distinto. Dentro de los argumentos que dan los/as trabajadores/as no capacitados/as para no aceptar el perfeccionamiento, están, por una parte, la ausencia de motivación, por otra, la escasa variedad de opciones y, finalmente, la falta de tiempo (OCDE, 2019). De no haber un cambio drástico y oportuno, la rapidez con que se está ejecutando la transformación de la dinámica laboral va a dejar fuera no solo a los/as actuales trabajadores/as, sino también a los/as actuales estudiantes. En relación a este punto, quisiera recordar la ley de Gordon Moore.

Solemos perder la perspectiva de lo que implica pensar en un crecimiento exponencial. Moore señaló en la década de los años 60 que el conocimiento tecnológico se duplicaría cada dos años. Eso significa que más de medio siglo después, la duplicación no es la misma. Es decir, la distancia que hay entre el conocimiento tecnológico y la estructura educativa tradicional es cada vez más abismante.

Entre 2004 y 2017 hubo un aumento en la necesidad de poseer las siguientes habilidades como requisito laboral: expresión escrita, razonamiento deductivo, razonamiento inductivo, comprensión oral y fluidez de ideas, en este mismo orden. Estas capacidades, lamentablemente, están determinadas por la calidad de la educación recibida 
desde la infancia. Con el paso del tiempo las brechas se van acumulando y llega un punto en el cual no es posible equilibrar el ejercicio laboral con el desarrollo de las habilidades exigidas para el desempeño, lo cual puede derivar en el despido de el/la trabajador/a.

Estas carencias en materia de formación educacional están asociadas a la clase social, puesto que, a menores recursos económicos, menores serán las opciones de optar a un colegio que pueda propiciar el desarrollo de capacidades de orden superior.

A los factores tradicionales que causan las brechas educativas, tales como la extracción social y la escolaridad, se les añade la edad. La incorporación de la tecnología, que pasó de ser un recurso opcional a un medio fundamental, deja a muchas personas en el camino. Pero esta vez no solo a estudiantes, sino también a profesionales cuya acción laboral no implicó necesariamente este tipo de conocimiento, hasta que los usos tecnológicos llegaron para imponerse.

\subsection{Innovaciones educativas y tecnología frente a la necesidad de la comunicación online}

Al alero del apoyo virtual, han surgido numerosos esfuerzos para crear plataformas educativas. Estas se dividen en comerciales, de software libre y de pago por uso de la nube.

Dentro de las comerciales, Blackboard está siendo usada por algunas universidades chilenas tales como la UNAB, UDLA y U. Mayor, entre otras. Las aplicaciones libres, como ATutor, Moodle, Sakai o Google Classroom, están disponibles online para ser usadas gratuitamente por numerosas instituciones del planeta. Nos encontramos frente a un mercado exponencial para uso educativo que la crisis de salud mundial ha puesto en evidencia.

Desde que en la década del 90 Gardner comenzó a hablar de las inteligencias múltiples, se abrió una puerta a la investigación basada en capacidades humanas que iban bastante más allá de lo meramente cognitivo. Sin embargo, tres décadas más tarde, esos estudios no trasuntan un cambio real en el sistema educativo. A partir de esa evidencia, es probable que este despertar del vínculo docente-estudiante establecido a través de la web sufra un 
cierto abandono una vez que se supere la crisis sanitaria. O sea, es posible que una vez más domine lo arcaico en oposición a lo emergente.

La abundante bibliografía surgida a partir de Howard Gardner comenzó a sumar una gran lista de autores que aportaron sus estudios y propuestas para desarrollar la inteligencia y la creatividad. Desde comienzos de siglo XXI, una gran cantidad de intelectuales y docentes comenzó a desarrollar estrategias al servicio de una mejor capacidad de aprendizaje. Hoy muchas de ellas son parte de libros de difusión internacional y también de libre disposición a través de internet.

Un caso muy interesante de tener en cuenta es la llamada "revolución del flipped classroom”. El año 2006, el profesor de química Jonathan Bergman creó la estrategia flipped classroom, a partir de un hecho puntual: había una cantidad importante de estudiantes que se ausentaban de las clases más importantes. El profesor estadounidense pensó que debía crear una estrategia para que todos/as sus estudiantes pudieran tener acceso por igual a la información que él quería entregar. A partir de esa inquietud, creó la clase invertida: la información llegaba al estudiantado a partir de un video de diez minutos, ellos debían responder preguntas y, luego, la clase presencial se establecía a partir de las respuestas y dudas que los/as estudiantes llevaban al aula.

Este recurso permitió usar el video, enviado a los correos de sus estudiantes, como un instrumento de aprendizaje tan efectivo que subió en un $25 \%$ el rendimiento de la clase. Hoy, en Estados Unidos y en Europa, el modelo flipped classroom se ha transformado en flipped learning. Es decir, emergió como un camino independiente de comunicación entre el/la profesor/a y el/la estudiante. Además, ha coexistido totalmente hermanado con la clase presencial. De hecho, en España, desde 2015, al alero de la organización de la Fundación BIAS, la Consejería de Educación de la Comunidad de Madrid realiza el Congreso Europeo de Flipped Classroom, donde llegan experiencias de todo el mundo para exponer y explorar cuáles son las variedades posibles. Bergman ha recibido numerosos premios y ha editado libros al respecto que han resultado de gran éxito en el mundo. Este profesor creó un recurso multimedial cuando no había obstáculos para realizar la clase presencial. Es decir, simplemente la pensó como un aporte y un complemento necesario en el contexto de la educación actual. Pero pareciera que hay una gran diferencia entre tener una educación presencial con la opción de un apoyo online y establecer todo el peso del vínculo pedagógico sobre una plataforma virtual. 


\section{Las variables de la educación online}

El factor que, aparentemente, más ha condicionado la aplicación del proceso de enseñanza-aprendizaje online ha sido la premura con la cual se ha efectuado. La extensión mundial de la pandemia de COVID-19 ha significado que todas las instituciones educativas (y también las empresas) han tenido que asumir un vínculo virtual para el cual eligieron una plataforma determinada. El hecho de que esta emergencia apareciera en Chile en el mes de marzo, obligó, en muchos casos, a improvisar una nueva forma de comunicación que puso en entredicho la capacidad de los/as usuarios/as para emplearla con eficiencia.

\subsection{Los/as docentes frente a la educación online}

Una vez que la necesidad del distanciamiento social se hizo imperativa, se establecieron los protocolos, tanto en la educación escolar como superior, para realizar las actividades educativas en modalidad online. No obstante, lo que ha ocurrido en la práctica es que los profesores han cambiado la pizarra por la pantalla, sin hacer mayor innovación en sus estrategias pedagógicas (Pérez, 2020).

En virtud de lo reciente del fenómeno, todavía no hay estudios, pero hay reportajes periodísticos que están dando cuenta del hecho. Los/as académicos/as expertos/as en educación a distancia han reparado en que los/as docentes no han sabido adaptar sus clases a esta nueva modalidad. De acuerdo a lo expresado por Natalia Ávila, jefa del programa de Doctorado en Educación de la Pontificia Universidad Católica de Chile:

Esto ha hecho que la teleeducación termine siendo un fiasco, porque lo que terminó ocurriendo, y es lo que he visto, es que los estudiantes están todo el día sentados frente a la pantalla en una clase tras otra de Zoom, y eso es lo que no debería haber pasado (Pérez, 2020).

De un modo similar reflexiona la docente Beatriz Fernández, académica del Centro de Investigación Avanzada en Educación de la Universidad de Chile, al referir su escepticismo a que en estos días se termine de consolidar la educación online, pero 
manifiesta su esperanza en un cambio en el trabajo docente: "Puede ser que tengamos más herramientas y recursos para trabajar que quizás antes no habíamos visualizado, porque estábamos centrados en lo presencial. Ahí hay una oportunidad, porque la escuela no ha cambiado mucho en los últimos 100 años” (Pérez, 2020).

Lo que expresan ambas académicas nos remite al concepto de enseñar que está arraigado en gran parte de los/as docentes: frontalidad y tradicionalidad. En ese sentido, la profesora Fernández manifiesta la esperanza de que la imperatividad del uso digital, con el tiempo, obligue a ver estos recursos desde una perspectiva más amigable.

Frente a estas situaciones, la modalidad de clase invertida de Jonathan Bergman emerge como un recurso extraordinariamente útil, puesto que desahoga la ansiedad del profesor que cree que debe estar todo el tiempo hablando al otro lado de la pantalla, y permite también descomprimir a los/as estudiantes, puesto que les otorga la libertad de acceder a los recursos en un tiempo determinado por ellos/as y las veces que quieran, para llegar a la clase a resolver sus inquietudes.

Mientras se espera que esto suceda, los/as estudiantes están sometidos a una presión inédita en la historia de la educación mundial. Sometidos/as al rigor de una pantalla y a la realidad de una internet saturada, que muchas veces se vuelve inestable. Todo ello sin considerar que estadísticamente hay un $12 \%$ de hogares chilenos que no tienen ese servicio (Injuv, 2019).

Mientras tanto, el avance tecnológico sigue su camino, IBM ya presentó en 2019 el IBM Q System One, un computador cuántico que posee una capacidad de velocidad de procesamiento millones de veces más rápida que los convencionales. Es decir, mientras más se tarde en incorporar los medios tecnológicos al aula, más se ampliará la brecha entre la educación tradicional y la vanguardia virtual a la que la juventud accede de manera natural. 


\section{Referencias}

Cadem. (abril, 2018). El Chile que viene. Recuperado de https://www.cadem.cl/encuestas/el-chile-que-viene-febrero-2019/

Dell Technologies/ Institute for the Future. (2017). Emerging Tecnologies' Impact on Society and Work in 2030. Recuperado de https://www.iftf.org/humanmachinepartnerships/

Foucault, M. (2018). Vigilar y castigar. Siglo XXI Editores. Buenos Aires.

García Canclini, N. (2001). Culturas híbridas. Estrategias para entrar y salir de la modernidad. Editorial Paidos. Buenos Aires.

Fábregas, M. (4 de diciembre de 2008). Pedro Echenique: "Cada dos años se duplica el conocimiento tecnológico". El País. Recuperado de https://elpais.com/diario/2008/12/05/galicia/1228475897_850215.html

Injuv. (2019). $9^{a}$ Encuesta Nacional de la Juventud, Santiago. Recuperado de http://www.injuv.gob.cl/noticias/9encuesta

OCDE. (2019). El futuro del trabajo. Perspectivas de empleo de la OCDE 2019. México: OCDE.

Pérez E., C. (10 de abril de 2020). El gran test de las clases "online". La Tercera. Recuperado de www.latercera.com/tendencias/noticia/el-gran-test-de-las-clasesonline/JOJOMO7S2BAB3FNRJYPPHGUZ3I/

Platt, A. (1997). La invención de la delincuencia. Siglo XXI Editores. México.

Rifkin, J. (2010). La civilización empática: la carrera hacia una conciencia global en un mundo en crisis. Paidós. Madrid.

Robinson, K. (2009). El elemento. Grijalbo. Barcelona.

Robinson, K. (2015). Escuelas creativas. Grijalbo. Barcelona.

Romero, S. (s/f). IBM presenta el primer ordenador cuántico de la historia. Muy Interesante. 
CLASES ONLINE O LA NECESIDAD DE ADAPTACIÓN A UNA NUEVA FORMA DE ESTABLECER EL PROCESO DE ENSEÑANZA-APRENDIZAJE / SOTO-AGUIRRE

https://www.muyinteresante.es/tecnologia/articulo/ibm-presenta-el-primerordenador-cuantico-comercial-de-la-historia-821547024277

Sandel, M. (2007). Contra la perfección. Marbot Ediciones. (2016). Buenos Aires.

Williams, R. (1980). Marxismo y Literatura. Península. Barcelona.

Zukerfeld, M. (2015). La tecnología en general, las digitales en particular: vida, milagros y familia de la "Ley de Moore". Revista Hipertextos, 2(4), 1-30. 